\title{
Solitary splenic metastasis of pulmonary carcinoid: A rare case report and literature review
}

\author{
TOSHIO NISHIKAWA, MASAHIKO TAKAHASHI, MASANOBU MORI, \\ YASUAKI KAMIKAWA and FUMIYUKI INOUE
}

Department of Thoracic Surgery, Inoue Hospital, Fukuyama, Hiroshima 721-0974, Japan

Received March 11, 2017; Accepted May 10, 2017

DOI: $10.3892 / \mathrm{mco} .2017 .1312$

\begin{abstract}
We herein report a rare case of solitary splenic metastasis of a pulmonary carcinoid, for which hand-assisted laparoscopic splenectomy was performed. A 73-year-old man was admitted to the Inoue Hospital (Fukuyama, Japan) in March 2015 with an abnormal shadow of the spleen. The patient had a history of right upper lobectomy with lymph node dissection for a pulmonary typical carcinoid (pT1N0M0 stage IA) 7 years earlier. Plain abdominal computed tomography (CT) revealed a low-density area in the spleen. Enhanced abdominal $\mathrm{CT}$ revealed a mass in the spleen measuring $3 \times 3 \mathrm{~cm}$. Blood tests and tumor marker analysis revealed no abnormalities. Abdominal magnetic resonance imaging revealed a mass that was low-intensity on T1- and high-intensity on T2-weighted images, and positron emission tomography revealed abnormal fluorodeoxyglucose accumulation in the mass. Hand-assisted laparoscopic splenectomy was performed for diagnosis and treatment. The resected specimen included a reddish-brown mass. On immunohistochemistry, the mass was positive for chromogranin A, synaptophysin and CD56, which was similar to the resected pulmonary carcinoid tumor; thus, splenic metastasis of pulmonary carcinoid was diagnosed. The postoperative course was uneventful and the patient is currently under observation and remains recurrence-free, with no adjuvant chemotherapy.
\end{abstract}

\section{Introduction}

Pulmonary carcinoids comprise $\sim 2 \%$ of all primary lung malignancies (1) and are considered to originate from pulmonary neuroendocrine cells. Pulmonary carcinoids are defined by their cytomorphology and neuroendocrine characteristics on histological examination, and immunohistochemical

Correspondence to: Dr Toshio Nishikawa, Department of Thoracic Surgery, Inoue Hospital, 3-23-46 Higashifukatsucho, Fukuyama, Hiroshima 721-0974, Japan

E-mail: gmd16091@s.okayama-u.ac.jp

Key words: pulmonary carcinoid, spleen, metastasis, splenectomy staining for chromogranin, synaptophysin, and/or neural cell adhesion molecule (CD56) (2). Typical carcinoids are distinguished from their atypical counterparts based on their mitotic rate and presence or absence of necrosis, and from tumorlets based on their size (2). While atypical carcinoids frequently metastasize to regional lymph nodes, liver, bone and brain (3), metastatic disease is rarely reported in typical carcinoids. Complete surgical resection for localized typical carcinoid is the standard treatment and is associated with a low recurrence rate (4-6). Patients undergoing surgical resection for typical carcinoids have excellent outcomes, with reported 5- and 10 -year survival rates of $85 \%$ (3). Although surgical resection for liver metastasis of pulmonary carcinoid may be considered with a curative intent, there is little evidence on the benefits of surgery for metastatic and recurrent disease.

Solitary splenic metastasis of solid tumors is rare. Splenic metastases from non-hematological malignancies are unusual, with an incidence of $0.6-1.1 \%$ in carcinoma populations (7). Carcinoid metastasis involving the spleen is extremely rare. Falk et al and Eriguchi et al reported splenic metastasis from ileal and gastric carcinoid tumors, respectively $(8,9)$. To the best of our knowledge, only three cases of splenic metastasis of pulmonary carcinoid have been reported to date; thus, solitary splenic metastasis of pulmonary carcinoid is rare (10-12). In the present case, splenic metastasis of pulmonary typical carcinoid was successfully treated by hand-assisted laparoscopic splenectomy (HALS). Laparoscopic splenectomy (LS) is currently considered to be the standard treatment for benign haematological disease, with low morbidity and mortality rates. However, there are very few reports of its efficacy for malignant disease of the spleen.

\section{Case report}

A 73-year-old man was admitted to the Inoue Hospital (Fukuyama, Japan) in March 2015 with an abnormal shadow of the spleen. The patient had a history of right upper lobectomy with lymph node dissection in 2008 for a primary typical carcinoid tumor of the lung, pathologically evaluated as pT1N0M0 stage IA. Plain abdominal computed tomography (CT) revealed a low-density area in the spleen, and enhanced abdominal CT revealed a mass measuring 3x3 cm (Fig. 1). Blood tests and tumor marker analysis revealed no abnormalities. On abdominal magnetic resonance imaging, the mass 
was low-intensity on T1- and high-intensity on T2-weighted images. Fluorodeoxyglucose (FDG)-positron emission tomography (PET) revealed abnormal FDG accumulation in the mass in the spleen (Fig. 2), but no accumulation in other tissues or organs. The tumor was considered to be a primary or metastatic splenic tumor; thus, HALS was performed in June 2015. Under general anesthesia, the patient was placed in the right semilateral decubitus position. A $10-\mathrm{cm}$ skin incision was made along the upper midline for a hand port and 3 ports were placed in the left upper quadrant of the abdomen. The gastrosplenic ligament and colonic attachments were divided, and the short gastric vessels were controlled with the LigaSure vessel sealing system (Medtronic Ltd., Dublin, Ireland) to allow isolation of the splenic hilum. Following mobilization of the spleen, the splenic hilar vessels were dissected and divided using a linear stapler. Following complete splenic mobilization, the spleen was placed in a bag and extracted through the upper midline incision. The total operative time was $155 \mathrm{~min}$ and the estimated intraoperative blood loss was only $10 \mathrm{ml}$. The resected spleen weighted $143 \mathrm{~g}$. The specimen included a tumor, measuring $3.6 \times 3.5 \mathrm{~cm}$, occupying the lower pole of the spleen. The tumor was reddish-brown in color, clearly demarcated from the adjacent splenic parenchyma, and exhibited no hemorrhage or necrosis. Histological analysis of the specimen confirmed the diagnosis of metastatic carcinoid tumor (chromogranin A-synaptophysin- and CD56-positive; absence of necrosis; mitotic count 0/high-power field; Ki-67 labelling index $2 \%$ ), with characteristics consistent with those of the primary pulmonary typical carcinoid (Fig. 3). The patient made a good postoperative recovery and currently remains recurrence-free, with no adjuvant therapy, 20 months after the operation (last follow-up, February 2017). The patient has consented to the publication of the case details and associated images.

\section{Discussion}

The spleen is a common site of secondary involvement by hematopoietic malignancies. By contrast, splenic metastasis of solid tumors is rare and is most often observed in skin melanoma and carcinomas of breast, lung, ovary, stomach and colorectum (7). The rarity of splenic metastasis may be explained by the following reasons: i) The poorly developed lymphoid system of the spleen prevents metastasis of tumor cells via the lymphatic route; ii) the sharp angle of the splenic artery branching from the celiac trunk prevents large clumps of tumor cells from passing through; and iii) large numbers of monocytes, immunoglobulin and opsonin synthesis and phagocytic activity protect against malignant cell implantation and proliferation (13). Solitary splenic metastasis without multivisceral disease is extremely rare (14). Satoh et al evaluated splenic metastasis from lung cancer (15); all cases exhibited accompanying metastasis to other organs, and it was concluded that a splenic mass accompanying metastasis to other abdominal organs in patients with a known lung cancer should be considered as a metastasis. Although typical carcinoids are associated with a low rate of metastasis, a splenic lesion accompanying metastasis to other abdominal organs in patients with a known tumor may be considered as a metastasis. Although splenic metastases have been found

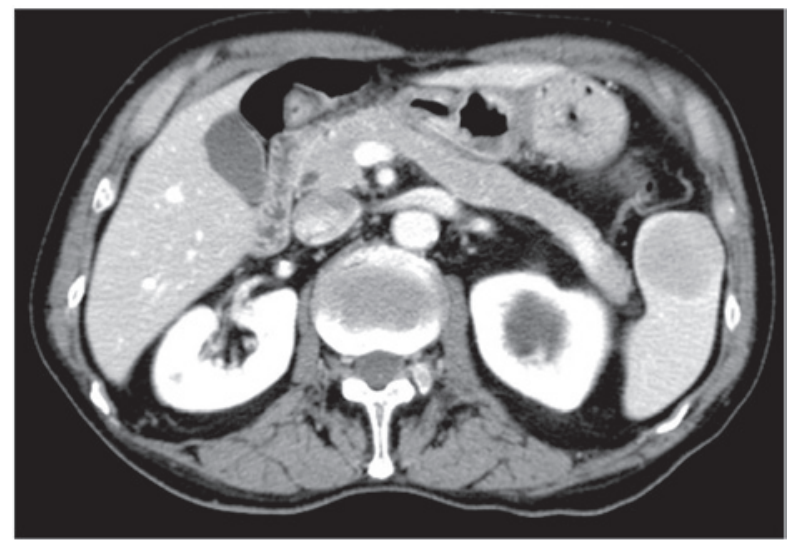

Figure 1. Enhanced abdominal computed tomography revealed a splenic mass measuring $3 \times 3 \mathrm{~cm}$.

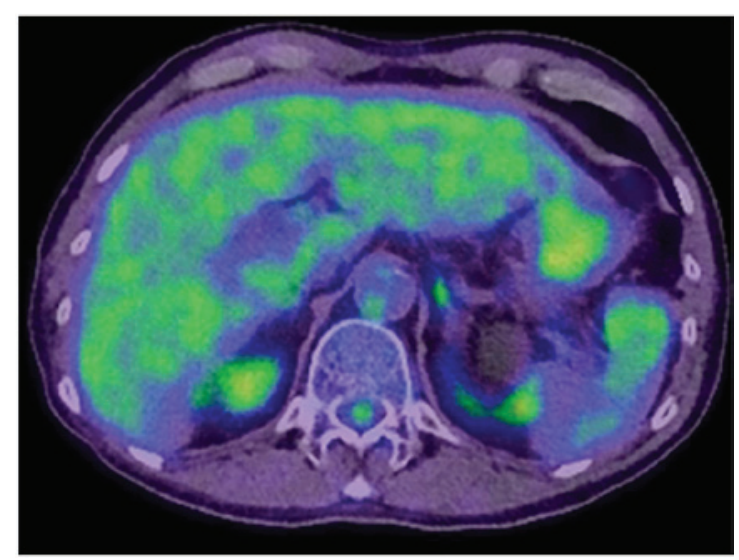

Figure 2. Fluorodeoxyglucose (FDG)-positron emission tomography revealed abnormal FDG accumulation in the splenic mass.

to arise more commonly from the left rather than the right lung (16), the splenic metastasis in the present case arose from a right-sided primary pulmonary carcinoid.

The majority of solitary splenic metastasis are asymptomatic; thus, they are often identified by ultrasonography or CT scan, as in the present case. A splenic metastasis should be suspected when an isolated splenic lesion is found during oncological follow-up. The findings of splenic metastasis ranged from a cystic lesion, low-density occupying lesion, to a solid mass, and exhibited different enhancement patterns on CT scan. ${ }^{18} \mathrm{~F}$-FDG PET is considered to be useful in distinguishing benign from malignant tumors of the spleen (17). In the present case, ${ }^{18} \mathrm{~F}$-FDG PET/CT revealed abnormal accumulation in the splenic tumor and was useful in diagnosing a malignant tumor in the spleen. The previous reports of solitary splenic metastasis from pulmonary carcinoid only include 3 cases. Gallium 68 whole-body PET was performed in only 1 case, but revealed no abnormal accumulation in the splenic metastasis. Lococo et al reported that Gallium 68-DOTA-peptide PET/CT was superior in detecting pulmonary typical carcinoid compared with ${ }^{18} \mathrm{~F}$-FDG PET/CT (18). Further investigation on the detection of splenic metastasis from pulmonary carcinoid with PET/CT is required. In the present case, ${ }^{18} \mathrm{~F}-\mathrm{FDG} \mathrm{PET} / \mathrm{CT}$ was useful for the confirmation of the absence of metastasis in the rest of the body, apart from the spleen. 
A

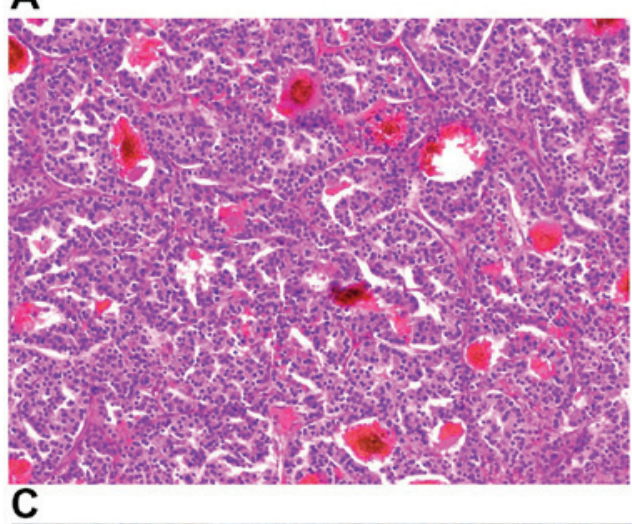

C

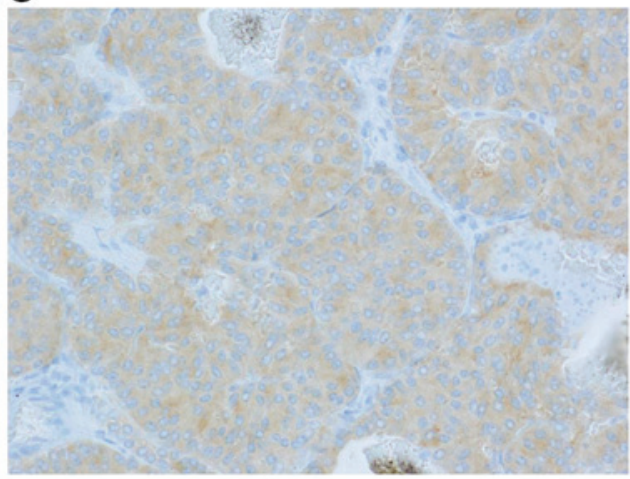

B

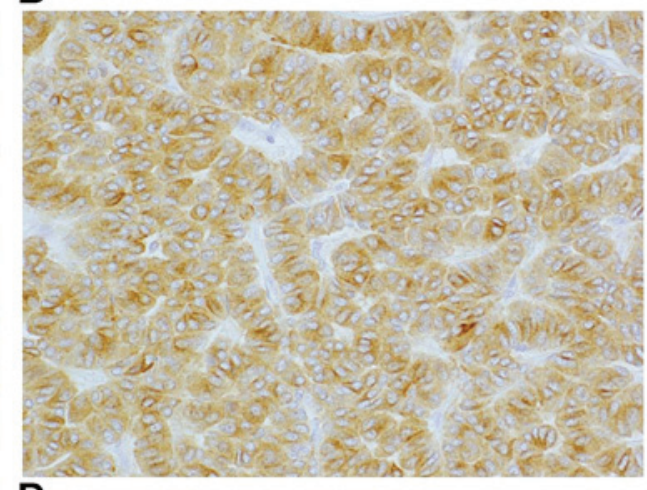

D

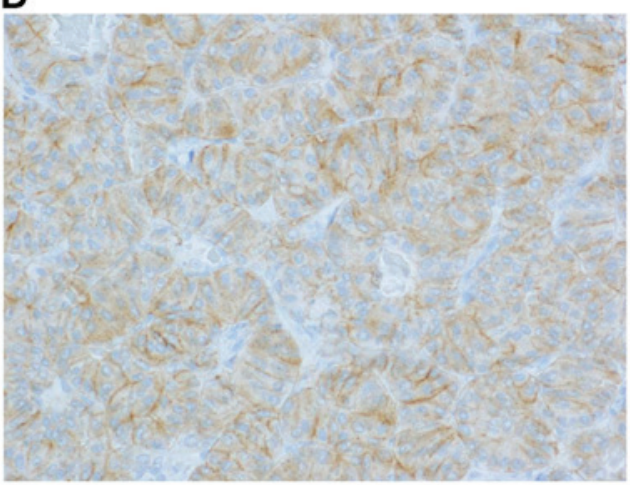

Figure 3. On histopathological examination, the resected splenic tumor resembled the resected carcinoid lung tumor; (A) hematoxylin and eosin staining (magnification, x20). On immunostaining, the tumor cells were positive for (B) chromogranin A, (C) synaptophysin and (D) CD56 (magnification, x40).

Splenectomy for metastatic splenic lesions provides a possible method of radical treatment and prevents further metastatic spread and complications due to the splenic tumor. In a review of 13 cases of splenic metastasis from lung carcinoma, Ando et al reported 6 patients who did not undergo splenectomy, 4 (67\%) of whom developed splenic rupture with a poor outcome (19). Although splenectomy provides the possibility of radical treatment for solitary splenic metastases, it is also important to confirm that the splenic metastasis it indeed solitary by PET/CT and/or other examinations. A splenic solitary metastasis may occasionally represent an initial clinical sign of whole-body metastasis. Under such conditions, surgical stress from splenectomy may cause adverse effects in the patient.

LS is the standard technique for removing spleens with benign disorders, and may also be applied for patients with malignant splenic disorders. Lopez et al reviewed previous reports of splenic metastasis treated by the open and laparoscopic approach, and concluded that the laparoscopic approach for splenic metastasis is a safe and reliable technique (20). To the best of our knowledge, this is the first report of a splenic metastasis of pulmonary carcinoid successfully treated by HALS. Altaf et al reported that the morbidity associated with HALS is comparable to that with LS, and concluded HALS is a viable alternative to open surgery in patients with sizeable spleens (21).

The benefits of the laparoscopic approach to splenic disorders are reduction in wound morbidity, shorter hospital stay and recovery time, and improved quality of life. The laparoscopic approach is particularly beneficial in cases of malignancy, as the associated shorter recovery times allow earlier introduction of chemotherapy. The HALS technique enables gentle retraction to obtain the required exposure. The hands may also be used to control unexpected bleeding that may otherwise be difficult to adequately expose or control. The main drawback of HALS mentioned in the literature is that it requires an additional incision for the placement of the hand port. Other theoretical disadvantages of the surgeon's hand in the abdomen include limiting the operative working space and predisposing to hand fatigue in long or complicated procedures (21). There is a lack of evidence for the use of HALS in the treatment of isolated malignant tumors of the spleen due to the rarity of this condition.

Based on the case presented herein, HALS is a useful approach for improving survival in patients with solitary splenic metastasis from pulmonary carcinoid.

\section{References}

1. Rekhtman N: Neuroendocrine tumors of the lung: An update. Arch Pathol Lab Med 134: 1628-1638, 2010.

2. Travis WD: Advances in neuroendocrine lung tumors. Ann Oncol 21 (Suppl 7): vii65-vii71, 2010.

3. Garcia-Yuste M, Matilla JM, Cueto A, Paniagua JM, Ramos G, Canizares MA and Muguruza I; Spanish Multi-centric Study of Neuroendocrine Tumours of the Lung for the Spanish Society of Pneumonology and Thoracic Surgery (EMETNE-SEPAR): Typical and atypical carcinoid tumours: Analysis of the experience of the Spanish multi-centric study of neuroendocrine tumours of the lung. Eur J Cardiothorac Surg 31: 192-197, 2007.

4. Cao C, Yan TD, Kennedy C, Hendel N, Bannon PG and McCaughan BC: Bronchopulmonary carcinoid tumors: Long-term outcomes after resection. Ann Thorac Surg 91: 339-343, 2011. 
5. Chen F, Sato T, Fujinaga T, Sakai H, Miyahara R, Bando T and Date H: Surgical management of bronchopulmonary typical carcinoid tumors: An institutional experience. Interact Cardiovasc Thorac Surg 11: 737-739, 2010.

6. Machuca TN, Cardoso PF, Camargo SM, Signori L, Andrade CF, Moreira AL, Moreira Jda S, Felicetti JC and Camargo JJ: Surgical treatment of bronchial carcinoid tumors: A single-center experience. Lung Cancer 70: 158-162, 2010.

7. Lam KY and Tang V: Metastatic tumors to the spleen: A 25-year clinicopathologic study. Arch Pathol Lab Med 124: 526-530, 2000.

8. Falk S and Stutte HJ: Splenic metastasis in an ileal carcinoid tumor. Pathol Res Pract 185: 238-244, 1989.

9. Eriguchi N, Aoyagi S, Hara M, Sakai T, Imayama H, Tanaka E, Hashimoto M, Imamura I and Kage M: Gastricente rochromaffin-like-cell tumor with liver and splenic metastases. J Gastroenterol 34: 383-386, 1999.

10. Takada T and Takami H: Solitary splenic metastasis of a carcinoid tumor of the lung eight years postoperatively. J Surg Oncol 67: 47-48, 1998.

11. Balmforth D, Skouras C, Palazzo F and Zacharakis E: Laparoscopic management for carcinoid metastasis to the spleen. HPB Surg 2011: 346507, 2011

12. Kaneko T, Kawano Y, Yasuno K, Inoue I, Tanaka H, Hasegawa A and Fukuda A: A case of splenic metastasis 8 years after radical resection of pulmonary carcinoid.J Jpn Surg Assoc 65: 3029-3032, 2004 (In Japanese).

13. Kim JC, Jeong CS, Kim HC, Yu CS, Kang GH and Lee MG: Isolated splenic metastasis from colorectal carcinoma: A case report. J Korean Med Sci 15: 355-358, 2000.
14. Compérat E, Bardier-Dupas A, Camparo P, Capron F and Charlotte F: Splenic metastases: Clinicopathologic presentation, differential diagnosis, and pathogenesis. Arch Pathol Lab Med 131: 965-969, 2007.

15. Satoh H, Watanabe K, Ishikawa H, Yamashita YT, Ohtsuka M and Sekizawa K: Splenic metastasis of lung cancer. Oncol Rep 8: 1239-1241, 2001.

16. Kinoshita A, Nakano M, Fukuda M, Kasai T, Suyama N, Inoue K, Nakata T, Shigematsu K, Oka M and Hara K: Splenic metastasis from lung cancer. Neth J Med 47: 219-223, 1995.

17. Metser U, Miller E, Kessler A, Lerman H, Lievshitz G, Oren R and Even-Sapir E: Solid splenic masses: Evaluation with 18F-FDG PET/CT. J Nucl Med 46: 52-59, 2005.

18. Lococo F, Perotti G, Cardillo G, De Waure C, Filice A, Graziano P, Rossi G, Sgarbi G, Stefanelli A, Giordano A, et al: Multicenter comparison of 18F-FDG and 68Ga-DOTA-peptide PET/CT for pulmonary carcinoid. Clin Nucl Med 40: e183-e189, 2015.

19. Ando K, Kaneko N, Yi L, Sato C, Yasui D, Inoue K, Misawa M and Ohkuni Y: Splenic metastasis of lung cancer. Nihon Kokyuki Gakkai Zasshi 47: 581-584, 2009 (In Japanese).

20. Lopez Monclova J, Targarona Soler E, Peraza Solis Y, Vidal Gonzalez P, Balague Ponz C, Rodriguez Luppi C and Trias Folch M: Laparoscopic approach for isolated splenic metastasis: Comprehensive literature review and report of 6 cases. Surg Laparosc Endosc Percutan Tech 23: 21-24, 2013.

21. Altaf AM, Ellsmere J, Jaap Bonjer H, El-Ghazaly TH and Klassen DR: Morbidity of hand-assisted laparoscopic splenectomy compared to conventional laparoscopic splenectomy: A 6-year review. Can J Surg 55: 227-232, 2012. 